\title{
Terrestrial Biodiversity and Climate Change
}

\author{
Mark A. Bradford and Robert J. Warren II \\ School of Forestry and Environmental Studies, Yale University, New Haven, CT 06511 \\ USA \\ mark.bradford@yale.edu, hexastylis@gmail.com
}

\begin{abstract}
Habitat change, invasive species, over-exploitation, pollution and climate change drive biodiversity loss. Together, these anthropogenic effects may have initiated the sixth mass extinction. Between $15-37 \%$ of terrestrial species may be lost by 2050 , and the remaining species likely will shift polewards and upwards to create novel assemblages of species. Reliable prediction of which species will go extinct, where they will relocate, and with whom they will associate, can only be achieved through substantial advances in our understanding of the physical and biological world. Indeed, many models associating species with climate change use oversimplified assumptions about the factors that regulate species survival, abundance and distribution. In reality, there are many biotic (e.g. competitors, predators, mutualists) and abiotic (e.g. temperature, moisture) factors that determine a species survival at micro- and macro-habitat scales. Current work typically focuses on a few abiotic factors at macro-habitat scales, leaving high uncertainty in how species will respond to climate change. The best management strategy for preserving biodiversity will, therefore, be a redress of the human footprint on the biosphere. Local initiatives alone may be ineffective. For example, wildlife reserves will be unsuccessful if climate change shifts temperature and rainfall outside the targeted species optimal requirements. If so, management must address whether the target species can migrate from the wildlife reserve toward suitable habitat. As such, conservation strategies must facilitate species movement that tracks favorable climate, via corridor maintenance and/or careful translocation of species to new habitat. The latter option is contentious given that movement of species might prevent extinction but threaten native species in the introduced range.
\end{abstract}




\section{Keywords}

Niche; Species distributions; Community composition; Extinction; Adaptation; Demography; Climate envelope models; Uncertainty; Forecasting; Prediction; Conservation; Species translocations

\section{Definition}

Extinction rates, and changes in the abundances and distributions of species, are accelerating under climate change. To predict and manage the consequences for biodiversity requires substantial advances in our fundamental understanding of the processes and factors that structure ecological communities.

\section{Key Information}

Nobel-prize winning physicist Richard Feynman, defined science as, “...the belief in the ignorance of experts." He meant that science, the body of knowledge about how the world works, must be re-evaluated before being accepted or rejected. It is through such judgment (observation and testing) that scientists hone scientific knowledge. No matter how advanced, this knowledge is always abounded at the edges by uncertainty. The uncertainty grows with the complexity of a problem, and complexity increases with the number of factors that might explain the problem. Our problem is explaining the distribution and abundance of a species, and those of the other species with which it is found (i.e. a community). Our confidence in the answers to this problem are low because many factors affect the distribution and abundance of species, and these factors likely change for each species in a community. Given uncertainty in which species will go extinct, where remaining species will occur, and what assortment of species future communities will contain, this chapter explores: (1) how climate change is affecting biodiversity; and (2) how we predict and might manage biodiversity change. 


\section{Evidence for Climate Change Effects on Biodiversity}

Terrestrial species are moving 11 meters per decade up elevation and 16.9 kilometers per decade poleward (Chen et al. 2011). Warming is driving these range shifts, but not all species can keep pace with a changing climate. Indeed, 15-37\% of terrestrial species may be 'committed to extinction' by 2050. Whereas 'background' extinction is not uncommon in the geologic record (of four billion known species, 99\% are extinct), generally species losses are balanced by the formation of new species. This balance is upset during mass extinctions: the loss of more than three-quarters of species in a geologically short interval. Only five known mass extinctions have occurred during Earth's history, and humans may have initiated a sixth (Barnosky et al. 2011). Approximately 100 species of birds, mammals and amphibians have been driven extinct in the last 100 years, and this extinction rate is expected to increase 10 to 100 times across this century (Millennium Ecosystem Assessment 2005).

The reason so many species will become extinct is not necessarily that climate is changing, but rather that climate is changing at an unprecedented rate. However, ice-core records indicate previous periods of equally rapid climatic change not associated with mass extinction (Hof et al. 2011). As such, the current extinction catalyst may be the interaction between broad-scale climate change and a human-modified landscape. For example, a road may block migration of a species as climate warms, condemning it to temperature conditions it cannot tolerate or immigrating species with which it cannot coexist (e.g. predators, competitors, parasites). Anthropogenic landscape modifications commonly are grouped into five categories: habitat change (e.g. urbanization), invasive species, over-exploitation (e.g. hunting), pollution (e.g. fertilizer run-off) and climate change. Each threat is context-dependent, both locally and at broad scales such as biomes (Millennium Ecosystem Assessment 2005). For example, temperate forest species in the eastern U.S. are unlikely to face the profound habitat change once caused by European settlers clearing land for agriculture, but the dense human population that today surrounds them threatens pollution and invasive species. The situation contrasts to that for species in tropical forest, where deforestation is the primary conservation concern. 
Given the complexity of species interactions and multiple drivers of biodiversity loss, it is not surprising that current climate responses are more complicated than 'upwards and polewards'. For example, warming in high-elevation forest can stimulate

tree growth that enhances shading to create suitable habitat for cold-tolerant understory herbs. In contrast, warming at lower elevation causes drier conditions that create habitat for drought-tolerant plants from warmer regions (Harrison et al. 2010). Such idiosyncrasies occur because species respond to multiple environmental factors, both biotic (e.g. tree growth modified the understory environment) and abiotic (e.g. waterlimitation, not direct temperature effects, selected drought-tolerant species and phenotypes). These examples emphasize that species respond to local conditions rather than mean global temperature. Yet uncertainty in climate projections grows exponentially when downscaling from global to seasonal patterns. This discrepancy generates two main uncertainty issues when predicting species responses to climate change: (1) species respond to local climate but it will require substantial advances in physical science to model local climate with certainty; and (2) species performance is regulated by multiple factors, and the control exerted by each factor varies with environment. This second issue falls in the realm of ecological science, and we explore it next.

\section{Predicting \& Managing Biodiversity in a Changing Climate}

Preventing species from going extinct is simple: ensure that births and deaths are at least equal. We introduce complexity and uncertainty when asking which factors regulate births and deaths, and hence cause species populations to increase, decrease or remain stable. Complexity increases further if we want to know how a population of a species will change in abundance and distribution at any one location. Populations often exist in networks (i.e. meta-populations) that influence each sub-population's abundance through migration. The abundance and phenotypic diversity of each population is the product of the meta-population's genetic diversity, migration rates and the local environment at each location. These dynamics create seemingly counterintuitive relationships between the abundance and distribution of populations and the environmental conditions where they occur. For example, immigration sustains a population even when the local environment 
is unfavorable (i.e. deaths exceed births), such as illustrated in Fig. 1a. These 'source-sink' dynamics illustrate limits to climate envelope models (CEMs), a common tool for projecting species responses to climate change. CEMs project species distributions from a climate envelope that is generated from observations of where species occur. These observations likely contain populations where deaths exceed births, and so are on the slow road to extirpation or maintained by dispersal; dynamics that CEM methods cannot elucidate. In other words, CEMs may project future distribution using data from populations that fall outside the set of environmental conditions under which a species can persist. These environmental conditions, or requirements for persistence, are defined as the niche, with habitat being the location where these requirements are met (Fig. 1).

At the broadest level, many predictive models of species distributions confuse presence with niche (Colwell and Rangel 2009). Refining this shortcoming requires models representing processes and factors influencing species persistence (Urban et al. 2011). Such models address five phenomena. (1) Interactions. Species interactions can be positive (e.g. mutualism) or negative (e.g. predation) and change in strength and nature as the environment varies. Loss of a pollinator might mean extirpation for a flowering plant whereas loss of a seed predator might allow population expansion. (2) Dispersal. Predictive models often assume species track shifting climates, yet migration may be impossible given habitat fragmentation, loss of a disperser, or simply the speed of climate change. (3) Mismatch between climate and distribution. Phenomena such as dispersallimitation mean there can be climates where a species could persist (i.e. within its niche) but where it does not occur. Equally, species occur under climate unsuitable for persistence, and they are maintained by dispersal. (4) Density-dependence. A species can limit itself more than its competitors do because high densities favor pathogens and intensify intraspecific resource competition. (5) Ecosystem engineering. Species can modify their environment to favor persistence, such as rhododendron trees do by producing compounds that bind soil-nitrogen to make it accessible only to them. These five phenomena mean species abundances and distributions under climate change can differ greatly from climate-only projections, and they remind us of a fundamental rule in scientific enquiry: pattern does not beget process. With this rule in mind, we still have to manage biodiversity in a changing climate. 
Species with small distributions, poor dispersal ability, narrow climate tolerances, low abundance and specialized habitat requirements, such as dependence on interspecific interactions, are especially vulnerable to climate change. Less vulnerable species might not go extinct, but changes in their abundance and distribution might alter the structure of communities and the ecosystem services they provide (e.g. food, clean water, firewood). The most effective solution to mitigating biodiversity loss is to redress environmental degradation. Realistically, policy and management will only limit the pace and extent of degradation, meaning communities will change. Robust ecological assessment and prediction of these changes will help us to adaptively manage ecosystem services in a changing climate. Habitat reserves are currently being used to prevent extinctions, but more research is needed to determine their effectiveness for current and future inhabitants. The possibility that climate change will make these reserves unsuitable has led to calls for assisted migration (mainly of short-range endemics) to suitable habitat (Thomas 2011). The idea is contentious given the many examples of deliberately- and accidentallyintroduced species that have become invasive. In such a debate we have to balance uncertainty over whether a species will go extinct versus uncertainty in whether it will become invasive. Moreover, no species is an island unto itself. We must identify key mutualists that may require translocation with the target species or assisted migration becomes a useless enterprise. The greatest challenge we have in maintaining terrestrial biodiversity under climate change is handling uncertainty in the rate and magnitude of local climate change, the ecological consequences, and how best to manage both.

There is no simple solution for preserving biodiversity under climate change. Yet it is clear that we will be powerless to assess and predict ecological responses to climate change if we focus only on correlations between species distributions and environmental factors. Climate change is certain: the Earth has changed, is changing and will change. How and why these changes impact biodiversity is uncertain, and it can only be determined by looking beyond species patterns and into the processes that drive them. 
a)

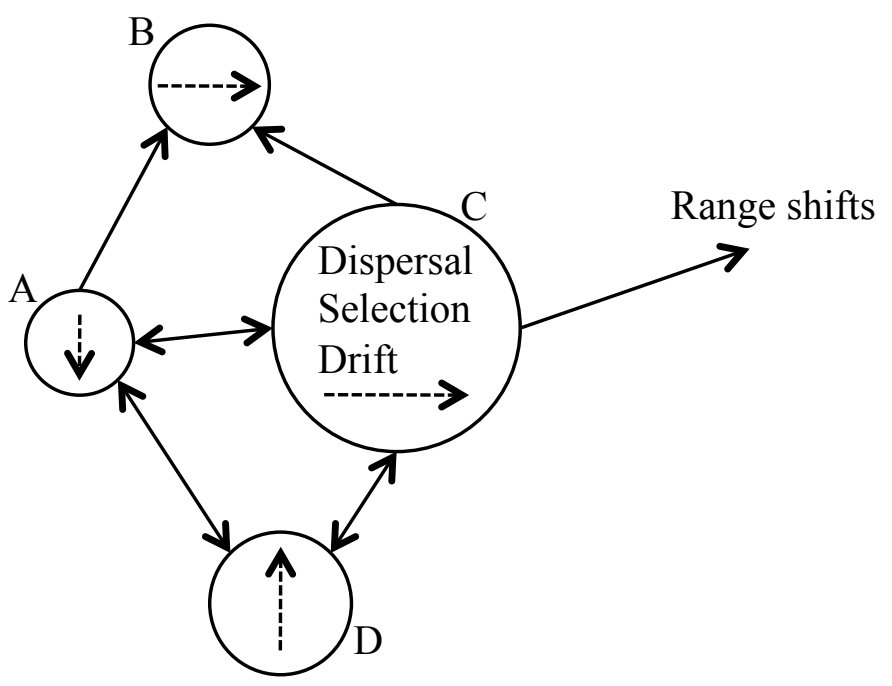

$\rightarrow--\rightarrow$ Directional change in population abundance

$\longrightarrow$ Direction of dispersal between populations

b)

Disease: leaf-spot fungi

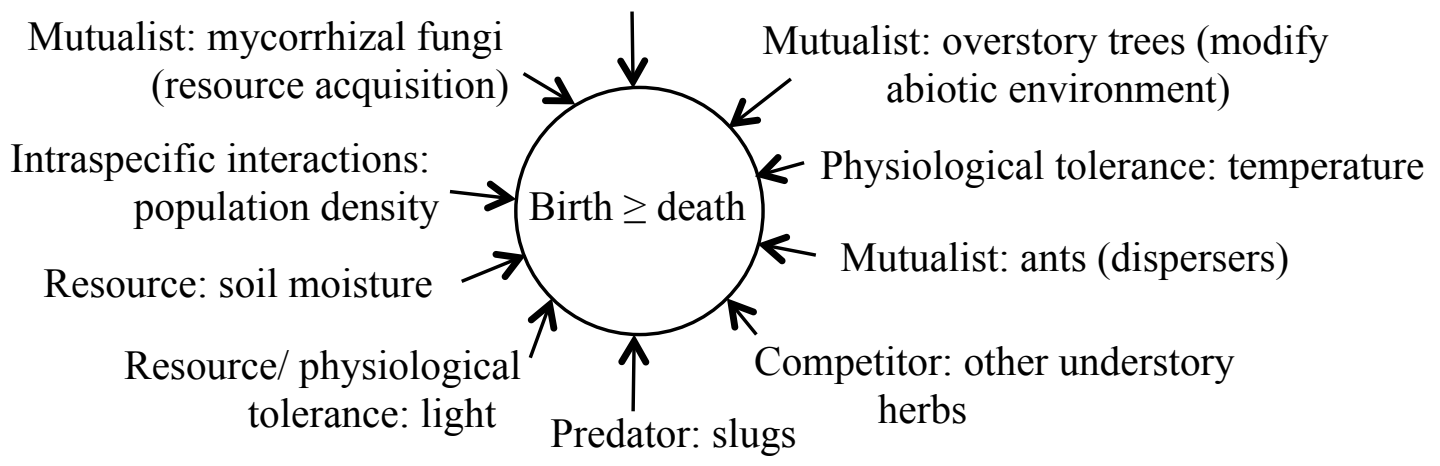

Figure 1. Three processes (a) and multiple factors (b) regulate the abundance and distribution of species, populations and communities. In (a) the three processes (dispersal, drift and selection) that determine population abundance and distribution, and hence community composition, are shown (following Vellend 2010). 'Speciation' also influences community composition but is omitted given that conservation efforts focus on preserving extant species. Dispersal is the movement of individuals in space and is essential if species are to find novel habitat (colonization) as climate changes (i.e. range shifts). Dispersal (immigration) can sustain populations when they exist in habitat unfavorable for persistence (e.g. Population B). Dispersal (emigration) also is essential 
within a population (e.g. Population C) to ensure local densities of related individuals remain below thresholds that promote disease epidemics, inbreeding depression and competition for resources. When different species disperse at different rates and distances, community composition will change. The other two processes regulating abundance are drift and selection. Given that mutation is random, change in genetic diversity of populations can be random (i.e. drift), as well as deterministic (i.e. selection). The same applies to abundances of species in communities. If species have similar birth, death, immigration and emigration rates, then species abundances in communities will change randomly. This phenomenon, known as ecological drift, introduces uncertainty into projection of how communities will respond to climate change. In contrast, ecological selection is deterministic. This means that understanding those factors that collectively influence selection should permit robust projections of species and community responses to changing environments. The factors influencing selection are biotic and abiotic, and examples are shown in (b) for an understory herb population. Collectively, these factors delineate species niche-requirements, which conceptually is the set of environmental conditions required for persistence (see Hutchinson 1978). The factors (or niche axes) together determine the $n$-dimensional niche shape (depicted here as a circle). Dispersers are shown as a factor but there is debate about whether dispersal is a niche axis. Importantly, the niche is a property of the species (or population) and so is not defined by the conditions regulating a population at any one point in space and time. Instead the set of environmental conditions, acting on a population that has demographic rates necessary for persistence, maps onto one point in niche space (Population C), or outside of niche space if death rates exceed births (Population B). This conception of the niche highlights why using known species distributions to project future ranges is fraught with uncertainty (see text).

\section{References}

Barnosky, A. D., N. Matzke, S. Tomiya, G. U. O. Wogan, B. Swartz, T. B. Quental, C. Marshall, J. L. McGuire, E. L. Lindsey, K. C. Maguire, B. Mersey, and E. A. 
Ferrer. 2011. Has the Earth's sixth mass extinction already arrived? Nature 471:51-57.

Chen, I.-C., J. K. Hill, R. Ohlemüller, D. B. Roy, and C. D. Thomas. 2011. Rapid range shifts of species associated with high levels of climate warming. Science 333:1024-1026.

Colwell, R. K. and T. F. Rangel. 2009. Hutchinson's duality: The once and future niche. Proceedings of the National Academy of Sciences 106:19651-19658.

Harrison, S., E. I. Damschen, and J. B. Grace. 2010. Ecological contingency in the effects of climatic warming on forest herb communities. Proceedings of the National Academy of Sciences 107:19362-19367.

Hof, C., I. Levinsky, M. B. Araújo, and C. Rahbek. 2011. Rethinking species' ability to cope with rapid climate change. Global Change Biology 17:2987-2990.

Hutchinson, G. E. 1978. An Introduction to Population Biology. Yale University Press, New Haven, CT.

Millennium Ecosystem Assessment. 2005. Ecosystems and Human Well-Being: Biodiversity Synthesis. World Resources Institute, Washington, DC.

Thomas, C. D. 2011. Translocation of species, climate change, and the end of trying to recreate past ecological communities. Trends in Ecology \& Evolution 26:216-221.

Urban, M. C., J. J. Tewksbury, and K. S. Sheldon. 2011. On a collision course: competition and dispersal differences create no-analogue communities and cause extinctions during climate change. Proceedings of the Royal Society of London B- Biological Sciences. DOI:10.1098/rspb.2011.2367.

Vellend, M. 2010. Conceptual synthesis in community ecology. The Quarterly Review of Biology 85:183-206.

\section{Cross-References}

Distribution of Terrestrial Ecosystems and Changes in Plant Community Composition

\section{Additional Recommended Reading}


Pulliam, H. R. 2000. On the relationship between niche and distribution. Ecology Letters 3:349-361.

Schmitz O. J. 2010. Resolving Ecosystem Complexity. Princeton Univ. Press, Princeton, NJ. 